\title{
Bisphenol-A Dose-dependently Regulates The Development of Ovary and Uterus through Differential Expression of METTL3
}

\section{Zhe Zhang}

State Key Laboratory of Analytical Chemistry for Life Science and Jiangsu Key Laboratory of Molecular Medicine, Medical School, Nanjing University, Nanjing 210093, China

\section{Yuan Zhu}

State Key Laboratory of Analytical Chemistry for Life Science and Jiangsu Key Laboratory of Molecular Medicine, Medical School, Nanjing University, Nanjing 210093, China

\section{Zhifan Zhang}

State Key Laboratory of Analytical Chemistry for Life Science and Jiangsu Key Laboratory of Molecular Medicine, Medical School, Nanjing University, Nanjing 210093, China

\section{Daojuan Wang}

State Key Laboratory of Analytical Chemistry for Life Science and Jiangsu Key Laboratory of Molecular Medicine, Medical School, Nanjing University, Nanjing 210093, China

\section{Hongwei Wang}

Nanjing University Medical School

\section{Guijun Yan}

Reproductive Medicine Center, the Affiliated Drum Tower Hospital of Nanjing University Medical School Yong Wang ( $\square$ yongwang@nju.edu.cn )

Nanjing University Medical School https://orcid.org/0000-0001-8366-7887

\section{Research}

Keywords: BPA, hyperandrogenism, METTL3, ovary, uterus

Posted Date: March 5th, 2020

DOI: https://doi.org/10.21203/rs.3.rs-16090/v1

License: (c) (1) This work is licensed under a Creative Commons Attribution 4.0 International License. Read Full License 


\section{Abstract}

Background: Environmental endocrine disruptors, which have a profound impact on the reproductive system, can cause endocrine and reproductive disorders, such as polycystic ovary syndrome (PCOS). Bisphenol-A (BPA) is a common endocrine disruptor which can affect the function of the reproductive system under low-dose conditions. However, the mechanism by which this molecule disrupts normal reproduction is still unclear. In this study, we investigated the effects of BPA on the RNA methyltransferase METTL3 in adolescent female rats which may be a possible mode of action of BPA.

Methods: 4-week-old Sprague-Dawley (SD) rats were intragastrically treated for 10 weeks, which were divided into blank group $(n=8)$, control group (soybean oil, $n=8)$, three BPA-treatment groups $(0.5 \mathrm{mg} / \mathrm{kg}$ BPA + soybean oil, $5 \mathrm{mg} / \mathrm{kg}$ BPA + soybean oil, $50 \mathrm{mg} / \mathrm{kg}$ BPA + soybean oil, $\mathrm{n}=8)$.

Results: The results showed that low-dose BPA $(0.5 \mathrm{mg} / \mathrm{kg})$ increased the coefficient of uteri and ovaries, and promoted the growth of uteri in rats without causing hyperandrogenism and ovarian polycystic changes. Oral BPA disturbed the expression of CYP17A1, CYP11A1 and METTL3 in ovaries and effected the level of serum testosterone.

Conclusions: Our results suggested that oral BPA might interfere uterine and ovarian morphology and the level of hormone with RNA methylation by disturbing the expression level of METTL3. RNA methylation will be a new way to explain the interference mechanism of BPA.

\section{Background}

Environmental endocrine disruptors (EEDs) are a group of exogenous substances which can interfere with the normal biological processes such as synthesis, secretion, transport and metabolism of natural hormones in an organism. EED can impact the functioning of nervous, immune and reproductive systems, resulting in endocrine diseases including polycystic ovary syndrome (PCOS). PCOS is a common endocrine disorder in women of childbearing age, characterized by sparse ovulation or anovulation, hyperandrogenism, and polycystic changes of the ovaries. Clinically, it is often characterized by menstrual disorders or amenorrhea, hairy, obesity, infertility, bilateral ovarian enlargement and cystic changes. Management of PCOS still focuses on resolving infertility, hyperandrogenism and polycystic ovaries [1, 2]. In recent years, researchers have tried to explore the etiology of PCOS from genetic, metabolic, environmental, and inflammatory perspectives [3]. Hyperandrogenism is one of the cardinal features of PCOS. Both clinical and laboratory studies have revealed that the over production of androgen is correlated to the expression of androgen synthases in the ovary [4, 5], such as CYP17, CYP11, StAR [6], HSD3B [7] and other proteases. It has been found that CYP11A1(tttta)n repeat polymorphism may be a potential molecular marker of PCOS risk $[8,9]$.

Being a very representative EED on endocrine disorders, Bisphenol-A (BPA) was first exploited as a synthetic estrogen. This substance is also commonly used to harden plastics. BPA in the environment can enter the human body through the mouth, skin, respiratory tract, etc. The bound free BPA can be 
metabolized by glucuronic acid or sulfonic acid in the liver and excreted via the kidney [10]. Fetal or perinatal BPA exposure can affect the brain [11], heart [12], liver [13], kidney [14], adipose tissue, breast $[15]$ and the reproductive system $[16,17]$ at lower relative concentrations, resulting in, for example, obesity, diabetes [18], PCOS [19]. Furthermore, substantial BPA-induced reproductive toxicity has been observed. BPA is found to be able to change the phenotypes of progeny by stably altering the parental epigenome [20]. Perinatal BPA exposure can alter the function of reproductive endocrine system by hypomethylating some imprinting genes during oocyte maturation or decreasing the expression of the estrogen receptor ER at both the mRNA and protein levels [21]. The direct toxicity of BPA is manifested in the disruption of decidualization in vitro [22], enhanced expression of estrogen receptors- $a(E R-a)$ and oxidative stress in embryonic stem cells [23], and promotion of apoptosis [24].

Clinical data demonstrate increased levels of BPA in patients with PCOS [25], and the levels can reach those in males [26]. PCOS induced by BPA exposure is also associated with hyperandrogenism and hyperinsulinism. BPA not only stimulates ovarian production of testosterone but also inhibits the activity of testosterone hydroxylase which helps maintain the testosterone concentration [27]; BPA-treated mice develop ovarian polycystic changes and luteal loss [28, 29], unbalanced expression between androgen synthesis-related enzymes and androgen receptors in the ovary, and abnormal expression of steroidogenic genes [30]. Following transient exposure to BPA before puberty, rats experience ovarian weight loss and follicle number decline [31]. Direct cellular exposure to BPA induces an increase in the percentage of germ cells and a decrease in the percentage of primordial follicles [32].

It is well established that epigenetic modification can regulate mRNA and protein expression without altering the DNA sequence. Several excellent reviews have alluded to the possible correlation of the hypomethylation status of gene promoter regions, such as EPHX1, to PCOS [33]. N6-methyladenosine (m6A), formed with methyltransferase, demethylase and YTHDF family proteins [34], is the most abundant regulator for the post-transcriptional modification of mammalian mRNA which is reversible. By using methylated RNA immunoprecipitation (MeRIP), the m6A residues are revealed to be located within a 100-200 nt-long transcript region. A strong correlation between the m6A and obesity, self-renewal and metastasis of stem cells [35], cell senescence [36], apoptosis [37] and metabolic regulation of cancer, e.g., gastric cancer [38] and colorectal cancer [39], has been reported. The interaction of RNA and protein in vivo affected by $\mathrm{m} 6 \mathrm{~A}$ has been intensively investigated, affecting the structure, maturation and stability of mRNA [40]. METTL3, highly conserved in most eukaryotes, is a major factor in abnormal m6A repair and cell self-renewal. Previous studies have indicated that increased levels of METTL3 and m6A are caused by the Wnt and PI3K-Akt pathway in gastric cancer [38], which is a detrimental factor in the prognosis of patients [41]. Furthermore, negative correlation between wide distribution of $\mathrm{m} 6 \mathrm{~A}$ and gene expression has been confirmed in chicken embryos, which coincides with the increase in m6A methylation peaks and their transcript levels during follicle selection [42], suggesting that $\mathrm{m} 6 \mathrm{~A}$ is critical in the occurrence of life. It is noteworthy that METTL3 can also suppress cell apoptosis by promoting IL$1 \beta$ production [43] and inhibit proliferation, migration and invasion of cancer cells through the p38/ERK pathway [39]. However, the biological functions of METTL3 await more thorough investigation. 
The effect of BPA on reproductive system has not been properly addressed in adolescent female rats. Moreover, RNA epigenetic modification has great impact in reproductive disorders [44]. Therefore, we underwent to further determine the effect of BPA on reproduction from an epigenetic modification perspective using PCOS as a model system to explore the mechanism of BPA-induced hyperandrogenism in adolescent female rats.

\section{Methods}

Animals

Experimental Sprague-Dawley (SD) rats, 3-week-old, weight $50 \mathrm{~g}$, were obtained from Qinglongshan, Inc., Nanjing, China. The animals were raised in SPF environment (Jiangsu Provincial Key Laboratory of Molecular Medicine), with temperature $22 \pm 1{ }^{\circ} \mathrm{C}$, relative humidity $50 \pm 1 \%$, light/dark cycle $12 / 12$ hours, providing free access to food and water.

After one-week adaptive feeding, the animals were randomly divided into 5 groups: blank group $(n=8)$, control group $(n=8)$, three BPA-treatment groups $(n=8)$. According to the previous studies, the lowest concentration of systemic toxicity caused by BPA is $500 \mathrm{mg} /(\mathrm{kg} \cdot \mathrm{d})$, p.o. [31], and the lowest concentration causing adverse reactions in the reproductive system is $50 \mathrm{mg} /(\mathrm{kg} \cdot \mathrm{d})$, p.o. [19]. BPA (obtained from Tianjin Guangfu Fine Chemicals Institute) was dissolved in absolute ethanol in a ratio of 1: 1 , configuring $4 \%$ solution after mixing it with soybean oil (obtained from Zhejiang Tianyushan medicinal oil limited Company). Set the BPA concentration gradient for three BPA-treatment groups gavaging daily with $0.5,5$ and $50 \mathrm{mg} /(\mathrm{kg} \cdot \mathrm{d}$ ) (Group BPA0.5, Group BPA5, Group BPA50) with 16-gauge needle at 16:00-18:00 according weight. Record the body weight and fur color daily. After 10 weeks, the rats were deeply anesthetized by $5 \%$ chloral hydrate with $0.07 \mathrm{~mL}$ per $10 \mathrm{~g}$ weight, i.p., after fasting overnight. The blood was collected from inferior vena cava, and livers, bilateral ovaries and uteri were dissected. Ovaries were fixed in $4 \%$ paraformaldehyde for $24 \mathrm{~h}$ at $4{ }^{\circ} \mathrm{C}$, and then embedded in paraffin. The rest of the tissues were frozen in $-80^{\circ} \mathrm{C}$ for further Western blotting.

Estrus Cycle

After 6-week gavaging when reproductive system of rats has been already mature, vaginal smear was taken from 7:30 to 8:00 every day. Moisten the dedicated cotton swab for gynecological examination with normal saline, and choose epithelial cells from $1 / 3$ lower segment of vagina and apply them on the slides. The cells were immersed in $1 \%$ toluidine blue dye solution for 30 minutes, and the back of the slide was rinsed with running water. After drying, the estrus cycle was described by cell type and morphology observed under the microscope (Leica Microsystems, Germany).

\section{Serum Testosterone Level}

Serum and red blood cells were separated after collecting the blood of rats with a coagulation tube, and the supernatant serum was taken after centrifugation. The serum testosterone level was measured according to the competition principle of the enzyme-linked immunosorbent assay (ELISA), using the Rat 
Testosterone ELISA Kit (obtained from Elabscience Biotechnology) to measure the optical density (OD value) of each well.

Hematoxylin And Eosin (he) Staining

The $4 \mu \mathrm{m}$-thick paraffin sections of the ovarian tissue were stained by hematoxylin and eosin after dewaxing. The sections were sealed by neutral gum after drying and observed under the optical microscope.

Western Blotting

Proteins of ovaries and livers were extracted using RIPA lysis buffer (Beyotime), determining concentration of protein by BCA working solution (Thermo Fisher). Total proteins of testes were extracted and electrophoresed on a SDS-polyacrylamide gel, and then transferred onto a PVDF membrane (Merck Millipore) with the electrophoresis \& trans-membrane system of Bio-Rad (Bio-Rad, USA). In this study, the target bands were sequentially incubated in primary antibodies, namely rabbit anti-Androgen receptor (1:500, Abcam, Cambridge, UK), rabbit anti-HSD3B1 (1:1,000, Bioss, Beijing, China), rabbit anti-StAR (1:500, Bioss), rabbit anti-METTL3 (1:1,000, Abcam), rabbit anti-CYP11A1 (1:1,000, Bioworld, Minnesota, USA), rabbit anti-CYP17A1 (1:500, Abcam), rabbit anti-GAPDH (1:5,000, Bioworld), rabbit anti-Estrogen receptor (1:1,000, Abcam). After incubating with secondary antibody (Goat-anti-rabbit lgG $(H+L) H R P)$ (Bioworld, USA), the target bands were analyzed with protein imprinted image collecting by the HRP-ECL chemiluminescence.

Data analysis

The target bands from Western blotting were analyzed using Image $\mathrm{J}$ on gray values. Data were expressed using mean \pm standard deviation (S.D.) or standard error of the mean (s.e.m.) from at least three independent experiments. Differences were analyzed using Graphpad prism 6.07 (Graphpad Inc.; La Jolla, USA), and statistical significance was determined using one-way analysis of variance (ANOVA). In this paper, statistical significance was set at a $\left.\mathrm{P}<0.05(*), \mathrm{P}<0.01{ }^{(\star *}\right)$ and $\left.\mathrm{P}<0.001{ }^{(\star \star \star}\right)$.

\section{Results}

Oral administration with low-dose BPA disrupts the morphology of the ovary and uterus.

To confirm whether BPA could stimulate unusual fat accumulation and growth by disrupting hormone balance, we recorded the body length, weight and fur color of rats daily during a 10-week-gavage treatment. No obvious differences were seen between BPA-treatment groups and control groups (Fig. 1a). Moreover, no weight differences between the blank group and the control group were observed, suggesting no discernable effect of soybean oil as a vehicle. This observation was consistent with no overwhelming liver anatomical changes (e.g., steatosis). Notably, a robust difference in rat ovary coefficient was observed between the BPA0.5 group and the BPA50 group (Fig. 1b). The uteri of rats after treatment with the BPA0.5 group expanded cystically, with colorless clear liquid inside (Fig. 1c). We next 
measured the length of uterine horn, and found extreme elongation after treatment with BPA, especially with a $0.5 \mathrm{mg} / \mathrm{kg}$ dose (Fig. 1d). We also observed increased uterus coefficient after treatment with BPA (Fig. 1e). The morphology of ovaries and corpus luteums of rats remained unchanged after treatment with BPA based on HE staining (Fig. 1f). To further examine whether follicular maturation was arrested at a certain stage, the number of follicles were progressively counted. Surprisingly, follicle numbers did not differ between treatment and control (Fig. 1g).

Oral administration with BPA extensively disrupts the estrus cycle.

We next investigated the functional impact of BPA on the physiology of the ovary and uterus. Rats vaginal smears were prepared 6 weeks after BPA treatment. We did not see the regular estrus cycles in rats of control group and BPA treatment groups. Interestingly, the intervals of diestrus and proestrus in a 10-day period was recorded. Shorter durations of proestrus after $5 \mathrm{mg} / \mathrm{kg}$ and $50 \mathrm{mg} / \mathrm{kg}$ treatments was recorded (Fig. 2a). However, durations of diestrus was not significantly affected by BPA treatment (Fig. 2b). Taken together, our data demonstrated that oral administration with BPA disrupted the normal patterns of vaginal epithelial cell growth in adolescent female rats.

Oral administration with low-dose BPA suppresses the expression of METTL3 without modulating hyperandrogenism.

We next asked whether the hormone level was regulated by oral administration of BPA. Modest changes in rat serum testosterone levels were observed after treatment with BPA. However, lower levels in the BPA50 group were observed compared with the BPA0.5 group (Fig. 3a). As regulation of hormonal responses can also be mediated by receptor modulation, we also measured the expression levels of androgen receptors and estrogen receptors in liver tissues. No statistically significant differences were observed (Fig. 3b-d). Taken together, our data suggested that oral low-dose BPA exposure did not seem to induce hyperandrogenism.

It has been previously reported that levels of androgen synthases were modulated by intraperitoneal injection of BPA [27]. We were interested in understanding whether oral low-dose exposure could also regulate androgen synthase production. We found that oral BPA treatment induced higher expression levels of the androgen synthase (Fig. 3e-j). Interestingly, we observed different levels of expression of METTL3 following treatment with BPA at different concentrations (Fig. 3e). The expression of METTL3 was progressively augmented with increasing concentrations of BPA (Fig. 3k).

\section{Discussion}

As a type of EEDs, BPA exerts agonistic or antagonistic effects depending on binding to different estrogen receptors in the nucleus [15]. It stimulates ovarian production of testosterone and inhibits the testosterone hydroxylase activity to increase testosterone levels. Several attempts have been made to show that the effect of low-dose BPA on female rats is more obvious than with a high concentration [27]. A strong relationship between BPA and obesity has been reported in the literatures, suggesting that early 
adipogenesis under perinatal exposure to BPA could be interfered with by regulating hypertrophy of fat cells and overexpression of fat genes. Nevertheless, the growth of rats in our study was normal without remarkable variation among different groups after a 10-week gavaging treatment, and no abnormal adipose tissues were found during the dissection. This result was in accord with studies on liver tissues without steatosis, suggesting that adolescent female rats had a stronger ability to metabolize exogenous BPA to avoid serious systemic toxicity than those young rats following exposure to BPA before they were born.

In our results, the tissue coefficient of uteri and ovaries following treatment with BPA at $0.5 \mathrm{mg} / \mathrm{kg}$ substantially changed, and abnormal histological changes of uteri were also recorded. Our findings supported differential effects following exposure using different concentrations of BPA, accompanied with the proliferation of uterine epithelial fibroblasts [16]. The state of endometrium and subsequent pregnancy preparation would be affected by changes in uterine morphology, which accounts for the mechanism of inference of uterus development by BPA. Other studies have pointed that irregular estrus cycles in adult rats are caused by high-dose BPA exposure, resulting in higher rate of rutting [19]. We observed that a more irregular estrus cycle following BPA treatment, especially in proestrus period.

Previous clinical and cellular studies have reported that BPA could reduce ovarian reserve function [45] and induce polycystic changes in the ovary [28]. Considerable research efforts have been devoted to hypomethylation of several imprinted genes caused by BPA-exposure during oocyte maturation. BPA can also accelerated the conversion from primordial follicles to primary follicles, and promote the degradation of primordial follicle pools [32], which provides evidences for ovarian ovulation interfered with by BPA. Our results showed integral follicles and corpus luteums without polycystic changes in ovarian sections in this study. The reason for failing to develop the polycystic model might be related to the first-pass effect of gavaging, which resulted in lower-dose BPA in rat blood because of its metabolism in the liver. Overall, the toxicity of oral BPA was not as strong as intraperitoneal injection, because of the higher threshold toxicity level through the oral route to induce ovarian polycystic changes.

Cellular studies have also revealed that BPA exerted anti-androgenic effects and increased activity of brain by stimulating ovarian production of testosterone, altering the splicing form of androgen receptor and increasing the thyroxin effect in the brain, which ultimately leads to hyperandrogenism [46]. In our study, there were no obvious differences of serum testosterone levels between the control group and the BPA-treatment groups, which indicated that oral BPA failed to cause hyperandrogenism. However, highdose BPA showed a stronger capacity in metabolizing androgen than low-dose BPA (Fig. 3a). The expression levels of proteins in the ovary and liver represented the ability of production and metabolism of androgen. The relative levels of CYP17A1, CYP11A1 and StAR in ovaries showed the differences between control group and BPA-treatment groups without inducing hyperandrogenism. Meanwhile, no differences were found on the levels of androgen receptor and estrogen receptor in the liver. Although BPA is reported to be capable of causing hepatocyte degeneration and necrosis by altering oxidative stress and affecting liver enzymes [47], our results failed to support any disorders in the liver. 
Interestingly, we found remarkable differences on the levels of METTL3 in BPA-treatment groups by Western blotting, which showed a relatively lower level in low-dose BPA and a higher level in high-dose BPA $(p<0.01)$. Several excellent reviews have described METTL3 as one of the most critical methyltransferases in RNA methylation, suggesting that its downregulation reflects internal changes in the m6A system. On the basis of our study, it could be concluded that these results might explain the interference mechanism of RNA involved in the reproductive system, although our results failed to directly confirm the effect of BPA on RNA methylation modification. The self-regulation of RNA methylation can stabilize the balance of methylation modification by various approaches such as methylation, demethylation and reduction of the demethylase activity.

Several questions still need to be addressed appropriately. For example, why does a lower level of serum testosterone and a higher level of METTL3 occurs simultaneously following treatment with BPA at $50 \mathrm{mg} / \mathrm{kg}$ ? Moreover, in order to gain more insight of the estrus cycle, will a shortened time interval before collecting vaginal smears reveal the duration of each period? All these questions require further experimental verification and explanation on the levels of $\mathrm{m} 6 \mathrm{~A}$, relevant proteins and hormones.

\section{Conclusions}

After gavage-treatment on adolescent female rats with different concentrations of BPA, our study demonstrated that oral BPA below a safe concentration failed to form ovarian polycystic changes or hyperandrogenism. On the contrary, low-dose BPA $(0.5 \mathrm{mg} / \mathrm{kg})$ interfered with abnormal uterine coefficient and the uterine morphology. BPA also disrupted the expression levels of CYP17A1 and CYP11A1 in ovaries and the levels of serum testosterone. However, the metabolic capacity of testosterone in adolescent female rats was stronger than younger rats with perinatal exposure. Our study provided the evidence on the differential expression of METTL3 by oral BPA in a dose-dependent manner for the first time. It suggested that the interference of BPA with the reproductive system in adolescent female rats could be explained by RNA methylation. Our data provide an alternative interpretation about the mechanisms that account for the effect of EEDs on the reproductive system.

\section{Abbreviations}

PCOS:polycystic ovary syndrome; BPA:Bisphenol-A; SD:Sprague-Dawley; EEDs:environmental endocrine disruptors; ER-a:estrogen receptors-a; m6A:N6-methyladenosine; MeRIP:methylated RNA immunoprecipitation; ELISA:enzyme-linked immunosorbent assay; HE:hematoxylin and eosin

\section{Declarations}

\section{Acknowledgments}

The authors are grateful to the help of Professor Xia zou for language.

\section{Author's contributions}


$Z Z, Z Y, Z Z F, W H W, Y G J$ and WY contributed to study design, analysis, method investigation and experiment performance. ZZ and WY writed the manuscript. ZZ, ZY, ZZF participated in the animal experiments and interpretation of the data. ZZ, ZY, ZZF and WY contributed to data acquisition and statistical analysis. All authors read and approved the final manuscript.

\section{Funding}

This work was supported by the National Natural Science Foundation of China [grant numbers 81971346, 81771539; and the Foundation of State Key Laboratory of Analytical Chemistry for Life Science [grant number 5431ZZXM1603].

\section{Availability of data and materials}

All data generated or analyzed during this study are included in this published article.

\section{Ethics approval and consent to participate}

All procedures of the animal experiments were approved by the Animal Research Committee of Nanjing University Medicine School.

\section{Consent for publication}

Not applicable

\section{Competing interests}

The authors declare that there are no competing interests associated with the manuscript.

\section{Author details}

${ }^{1}$ State Key Laboratory of Analytical Chemistry for Life Science and Jiangsu Key Laboratory of Molecular Medicine, Medical School, Nanjing University, Nanjing 210093, China; ${ }^{2}$ Reproductive Medicine Center, the Affiliated Drum Tower Hospital of Nanjing University Medical School, Nanjing 210008, China

\section{References}

1. Cassidy-Vu L, Joe E, Kirk JK. Role of Statin Drugs for Polycystic Ovary Syndrome. Journal of family \& reproductive health. 2016; 10: 165-75.

2. Balen AH, Morley LC, Misso M, Franks S, Legro RS, Wijeyaratne CN, et al. The management of anovulatory infertility in women with polycystic ovary syndrome: an analysis of the evidence to support the development of global WHO guidance. Human reproduction update. 2016; 22: 687-708.

3. De Leo V, Musacchio MC, Cappelli V, Massaro MG, Morgante G, Petraglia F. Genetic, hormonal and metabolic aspects of PCOS: an update. Reproductive biology and endocrinology : RB\&E. 2016; 14: 38. 
4. Wu C, Wei K, Jiang Z. 5alpha-reductase activity in women with polycystic ovary syndrome: a systematic review and meta-analysis. Reproductive biology and endocrinology : RB\&E. 2017; 15: 21.

5. Walters KA, Handelsman DJ. Role of androgens in the ovary. Molecular \& Cellular Endocrinology. 2017: S0303720717303532.

6. Hasegawa T, Kamada Y, Hosoya T, Fujita S, Nishiyama Y, Iwata N, et al. A regulatory role of androgen in ovarian steroidogenesis by rat granulosa cells. The Journal of steroid biochemistry and molecular biology. 2017; 172: 160-5.

7. Wang L, Li S, Zhao A, Tao T, Mao X, Zhang P, et al. The expression of sex steroid synthesis and inactivation enzymes in subcutaneous adipose tissue of PCOS patients. The Journal of steroid biochemistry and molecular biology. 2012; 132: 120-6.

8. Shen W, Li T, Hu Y, Liu H, Song M. Common polymorphisms in the CYP1A1 and CYP11A1 genes and polycystic ovary syndrome risk: a meta-analysis and meta-regression. Archives of gynecology and obstetrics. 2014; 289: 107-18.

9. Reddy KR, Deepika ML, Supriya K, Latha KP, Rao SS, Rani VU, et al. CYP11A1 microsatellite (tttta)n polymorphism in PCOS women from South India. Journal of assisted reproduction and genetics. 2014; 31: 857-63.

10. Fenichel P, Chevalier N, Brucker-Davis F. Bisphenol A: an endocrine and metabolic disruptor. Annales d'endocrinologie. 2013; 74: 211-20.

11. Khurana S, Ranmal S, Ben-Jonathan N. Exposure of newborn male and female rats to environmental estrogens: delayed and sustained hyperprolactinemia and alterations in estrogen receptor expression. Endocrinology. 2000; 141: 4512-7.

12. Ola-Davies OE, Olukole SG. Gallic acid protects against bisphenol A-induced alterations in the cardiorenal system of Wistar rats through the antioxidant defense mechanism. Biomedicine \& Pharmacotherapy.

13. Amraoui W, Adjabi N, Bououza F, Boumendjel M, Taibi F, Boumendjel A, et al. Modulatory Role of Selenium and Vitamin E, Natural Antioxidants, against Bisphenol A-Induced Oxidative Stress in Wistar Albinos Rats. Toxicological research. 2018; 34: 231-9.

14. Yuan J, Kong Y, Ommati MM, Tang Z, Li H, Li L, et al. Bisphenol A-induced apoptosis, oxidative stress and DNA damage in cultured rhesus monkey embryo renal epithelial Marc-145cells. Chemosphere. 2019; 234: 682-9.

15. Seachrist DD, Bonk KW, Ho SM, Prins GS, Soto AM, Keri RA. A review of the carcinogenic potential of bisphenol A. Reproductive toxicology (Elmsford, NY). 2016; 59: 167-82.

16. Li Q, Davila J, Kannan A, Flaws JA, Bagchi MK, Bagchi IC. Chronic Exposure to Bisphenol A Affects Uterine Function During Early Pregnancy in Mice. Endocrinology. 2016; 157: 1764-74.

17. Tomza-Marciniak A, Stepkowska P, Kuba J, Pilarczyk B. Effect of bisphenol A on reproductive processes: A review of in vitro, in vivo and epidemiological studies. Journal of applied toxicology : JAT. 2018; 38: 51-80. 
18. Martinez-Pinna J, Marroqui L, Hmadcha A, Lopez-Beas J, Soriano S, Villar-Pazos S, et al. Oestrogen receptor beta mediates the actions of bisphenol-A on ion channel expression in mouse pancreatic beta cells. Diabetologia. 2019; 62: 1667-80.

19. Fernandez M, Bourguignon N, Lux-Lantos V, Libertun C. Neonatal exposure to bisphenol a and reproductive and endocrine alterations resembling the polycystic ovarian syndrome in adult rats. Environmental health perspectives. 2010; 118: 1217-22.

20. Dolinoy DC, Huang D, Jirtle RL. Maternal nutrient supplementation counteracts bisphenol A-induced DNA hypomethylation in early development. Proceedings of the National Academy of Sciences of the United States of America. 2007; 104: 13056-61.

21. Chao HH, Zhang XF, Chen B, Pan B, Zhang LJ, Li L, et al. Bisphenol A exposure modifies methylation of imprinted genes in mouse oocytes via the estrogen receptor signaling pathway. Histochemistry and cell biology. 2012; 137: 249-59.

22. Olson MR, Su R, Flaws JA, Fazleabas AT. Bisphenol A impairs decidualization of human uterine stromal fibroblasts. Reproductive toxicology (Elmsford, NY). 2017; 73: 339-44.

23. Cho YJ, Park SB, Park JW, Oh SR, Han M. Bisphenol A modulates inflammation and proliferation pathway in human endometrial stromal cells by inducing oxidative stress. Reproductive toxicology (Elmsford, NY). 2018; 81: 41-9.

24. Su Y, Quan C, Li X, Shi Y, Duan P, Yang K. Mutual promotion of apoptosis and autophagy in prepubertal rat testes induced by joint exposure of bisphenol $A$ and nonylphenol. Environmental pollution (Barking, Essex : 1987). 2018; 243: 693-702.

25. Palioura E, Diamanti-Kandarakis E. Polycystic ovary syndrome (PCOS) and endocrine disrupting chemicals (EDCs). Reviews in endocrine \& metabolic disorders. 2015; 16: 365-71.

26. Takeuchi T, Tsutsumi 0 . Serum bisphenol a concentrations showed gender differences, possibly linked to androgen levels. Biochemical and biophysical research communications. 2002; 291: 76-8.

27. Huo X, Chen D, He Y, Zhu W, Zhou W, Zhang J. Bisphenol-A and Female Infertility: A Possible Role of Gene-Environment Interactions. International journal of environmental research and public health. 2015; 12: 11101-16.

28. Kato H, Ota T, Furuhashi T, Ohta Y, Iguchi T. Changes in reproductive organs of female rats treated with bisphenol A during the neonatal period. Reproductive toxicology (Elmsford, NY). 2003; 17: 2838.

29. Nikaido Y, Danbara N, Tsujita-Kyutoku M, Yuri T, Uehara N, Tsubura A. Effects of prepubertal exposure to xenoestrogen on development of estrogen target organs in female CD-1 mice. In vivo (Athens, Greece). 2005; 19: 487-94.

30. Santamaria C, Durando M, Munoz de Toro M, Luque EH, Rodriguez HA. Ovarian dysfunctions in adult female rat offspring born to mothers perinatally exposed to low doses of bisphenol A. The Journal of steroid biochemistry and molecular biology. 2016; 158: 220-30.

31. Li Y, Zhang W, Liu J, Wang W, Li H, Zhu J, et al. Prepubertal bisphenol A exposure interferes with ovarian follicle development and its relevant gene expression. Reproductive toxicology (Elmsford, 
NY). 2014; 44: 33-40.

32. Zhou C, Wang W, Peretz J, Flaws JA. Bisphenol A exposure inhibits germ cell nest breakdown by reducing apoptosis in cultured neonatal mouse ovaries. Reproductive toxicology (Elmsford, NY). 2015; 57: 87-99.

33. Sang Q, Li X, Wang H, Wang H, Zhang S, Feng R, et al. Quantitative methylation level of the EPHX1 promoter in peripheral blood DNA is associated with polycystic ovary syndrome. PloS one. 2014; 9 : e88013.

34. Maity A, Das B. N6-methyladenosine modification in mRNA: machinery, function and implications for health and diseases. The FEBS journal. 2016; 283: 1607-30.

35. Niu Y, Wan A, Lin Z, Lu X, Wan G. N (6)-Methyladenosine modification: a novel pharmacological target for anti-cancer drug development. Acta pharmaceutica Sinica B. 2018; 8: 833-43.

36. Casella G, Tsitsipatis D, Abdelmohsen K, Gorospe M. mRNA methylation in cell senescence. Wiley interdisciplinary reviews RNA. 2019: e1547.

37. Dominissini D, Moshitch-Moshkovitz S, Schwartz S, Salmon-Divon M, Ungar L, Osenberg S, et al. Topology of the human and mouse m6A RNA methylomes revealed by m6A-seq. Nature. 2012; 485: 201-6.

38. Zhang C, Zhang M, Ge S, Huang W, Lin X, Gao J, et al. Reduced m6A modification predicts malignant phenotypes and augmented Wnt/PI3K-Akt signaling in gastric cancer. Cancer medicine. 2019; 8: 4766-81.

39. Deng R, Cheng Y, Ye S, Zhang J, Huang R, Li P, et al. m(6)A methyltransferase METTL3 suppresses colorectal cancer proliferation and migration through p38/ERK pathways. OncoTargets and therapy. 2019; 12: 4391-402.

40. Liu N, Zhou KI, Parisien M, Dai Q, Diatchenko L, Pan T. N6-methyladenosine alters RNA structure to regulate binding of a low-complexity protein. Nucleic acids research. 2017; 45: 6051-63.

41. Liu T, Yang S, Sui J, Xu SY, Cheng YP, Shen B, et al. Dysregulated N6-methyladenosine methylation writer METTL3 contributes to the proliferation and migration of gastric cancer. Journal of cellular physiology. 2019.

42. Fan Y, Zhang C, Zhu G. Profiling of RNA N6-methyladenosine methylation during follicle selection in chicken ovary. Poultry science. 2019.

43. Liu Q, Li M, Jiang L, Jiang R, Fu B. METTL3 promotes experimental osteoarthritis development by regulating inflammatory response and apoptosis in chondrocyte. Biochemical and biophysical research communications. 2019; 516: 22-7.

44. Pau CT, Mosbruger T, Saxena R, Welt CK. Phenotype and Tissue Expression as a Function of Genetic Risk in Polycystic Ovary Syndrome. PloS one. 2017; 12: e0168870.

45. Zhou W, Fang F, Zhu W, Chen ZJ, Du Y, Zhang J. Bisphenol A and Ovarian Reserve among Infertile Women with Polycystic Ovarian Syndrome. International journal of environmental research and public health. 2016; 14. 
46. Acconcia F, Pallottini V, Marino M. Molecular Mechanisms of Action of BPA. Dose-response : a publication of International Hormesis Society. 2015; 13: 1559325815610582.

47. Khan S, Beigh S, Chaudhari BP, Sharma S, Aliul Hasan Abdi S, Ahmad S, et al. Mitochondrial dysfunction induced by Bisphenol $A$ is a factor of its hepatotoxicity in rats. Environmental toxicology. 2016; 31: 1922-34.

\section{Figures}


a

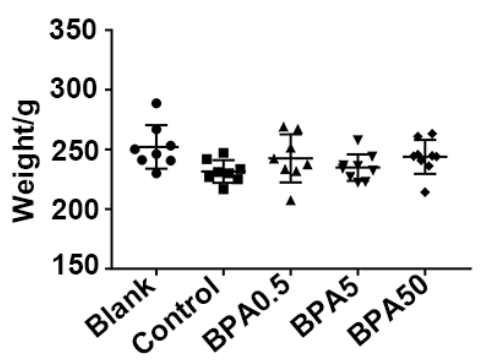

b

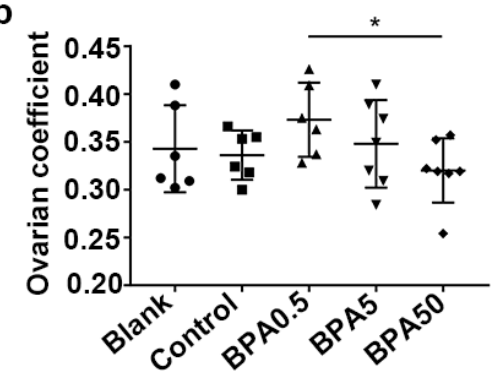

C

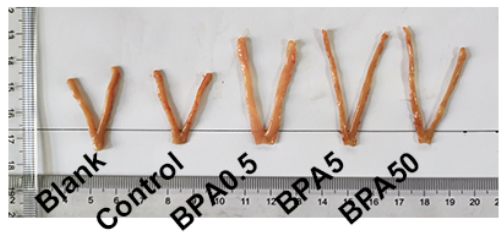

d E

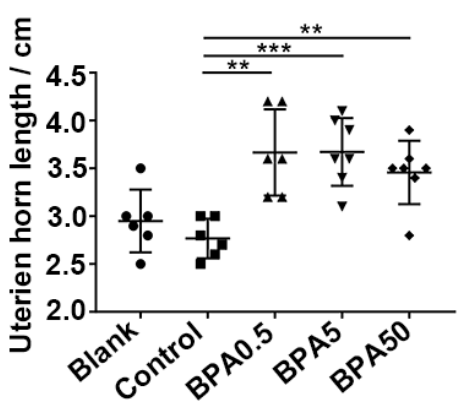

e

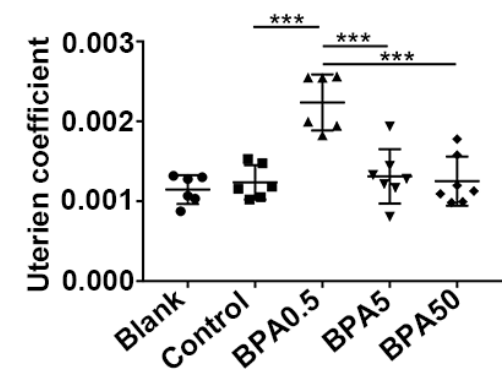

f
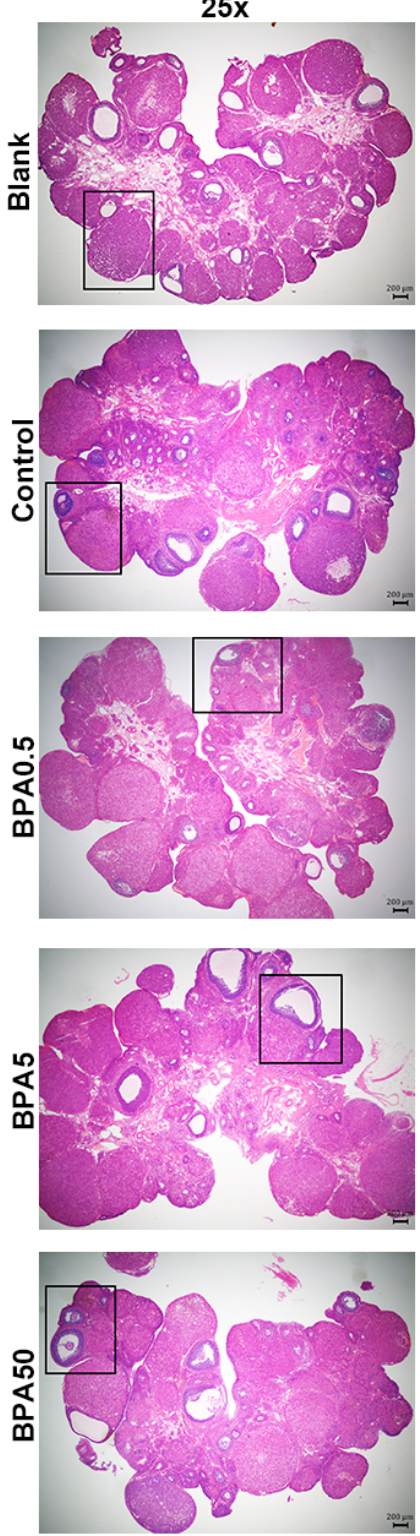

g

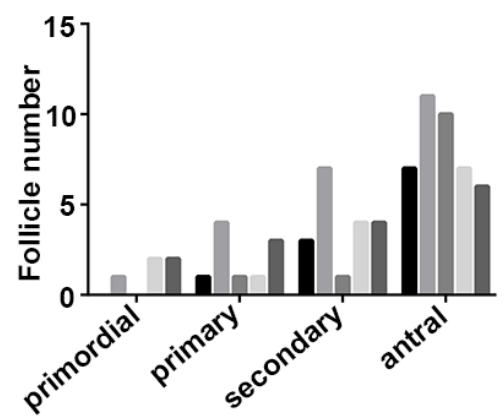

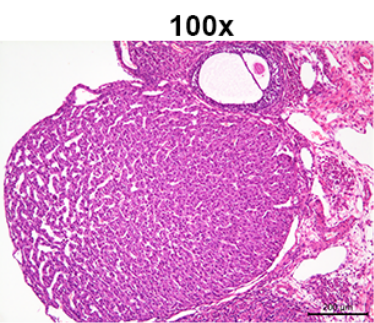
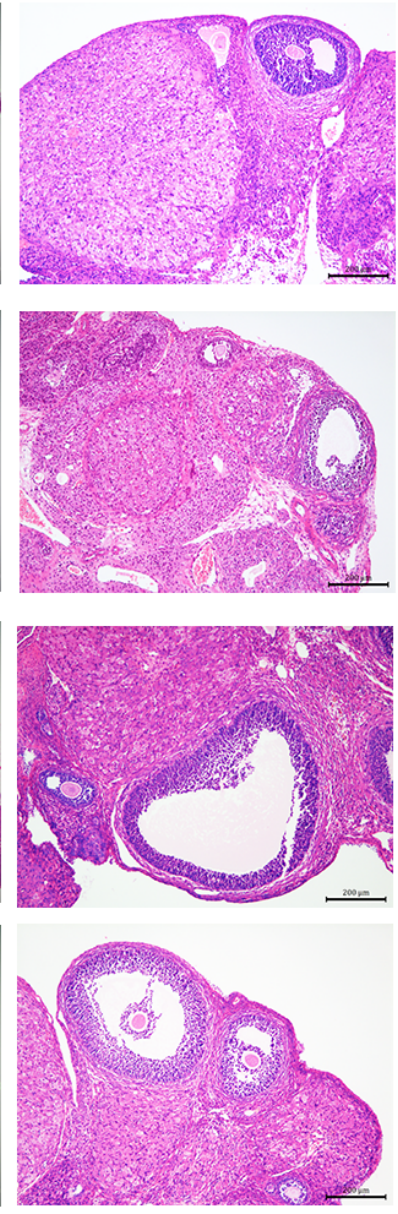

- Blank

- Control

BPA0.5

BPA5

- BPA50

\section{Figure 1}

Low-dose of Bisphenol-A (BPA) disrupts the morphology of uterine and ovarian tissues. Rats received daily gavaging with BPA for 10 consecutive days. a Rat body weights were recorded at the last treatment day. $b$ Ovaries were harvested and weighed to calculate the ovarian coefficient. * $P<0.05$. c Uterine tissues were cleared of surrounding connective tissues to contrast the morphology of uterus. $d$ The actual length of uterine horn was measured. ${ }^{* *} \mathrm{P}<0.01$, ${ }^{* *} \mathrm{P}<0.001$. e Uteri were harvested and weighed to 
calculate the coefficient. $* \star \star P<0.001$. $f$ Paraffin sections of the ovarian tissues were stained by hematoxylin and eosin (HE) followed by microscopic examination at $25 \mathrm{x}$; the insets show $100 \mathrm{x} . \mathrm{g}$ Follicle numbers were enumerated at various developmental stages.
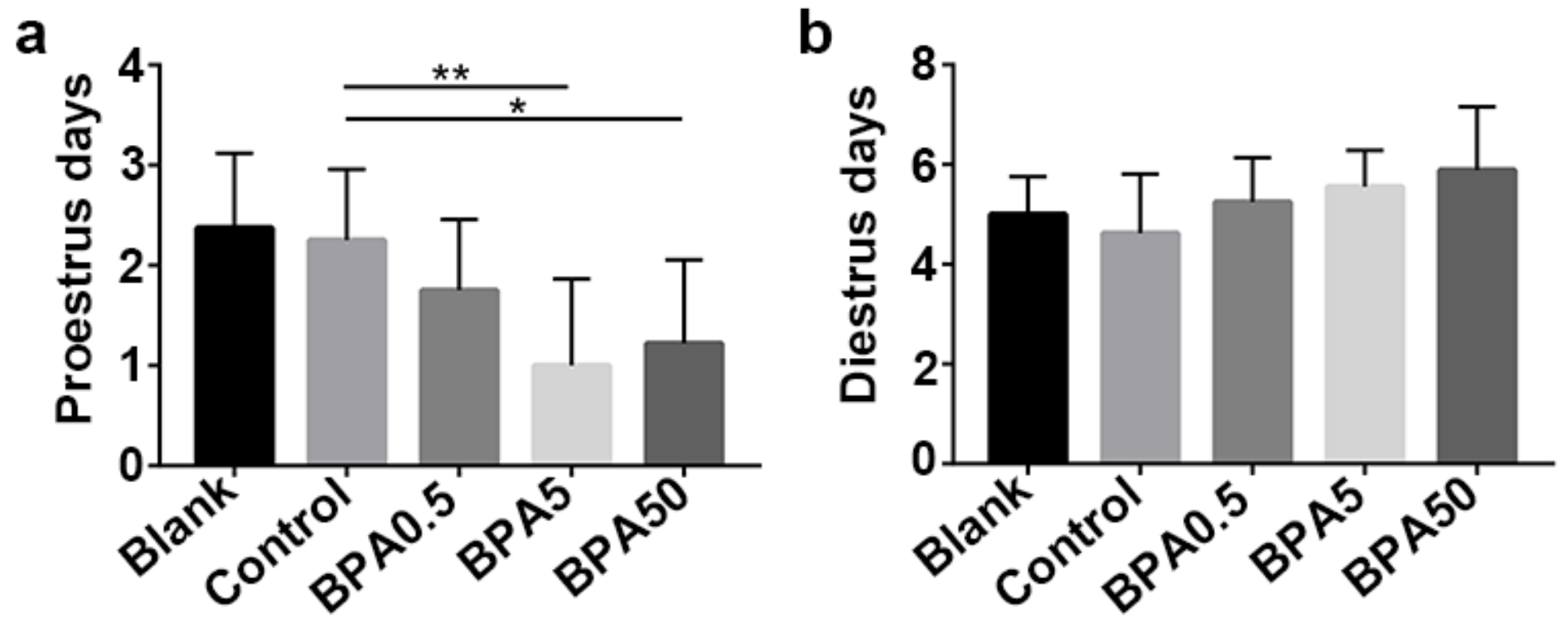

Figure 2

Oral Bisphenol-A (BPA) disrupts the estrus cycle. Rats received daily gavaging with BPA for 10 consecutive days. Daily vaginal smears were taken since week 6 after the BPA treatment and were immersed in a $1 \%$ toluidine blue solution to reveal the estrus cycle. a Variation in proestrus periods during a 10-day estrus cycle was recorded. ${ }^{*} \mathrm{P}<0.05,{ }^{*} \mathrm{P}<0.01$. b Variation in diestrus periods during a 10-day estrus cycle was recorded. 
a

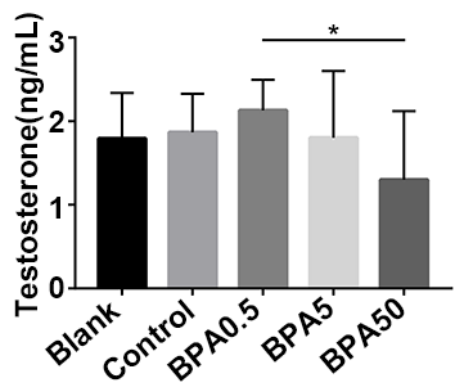

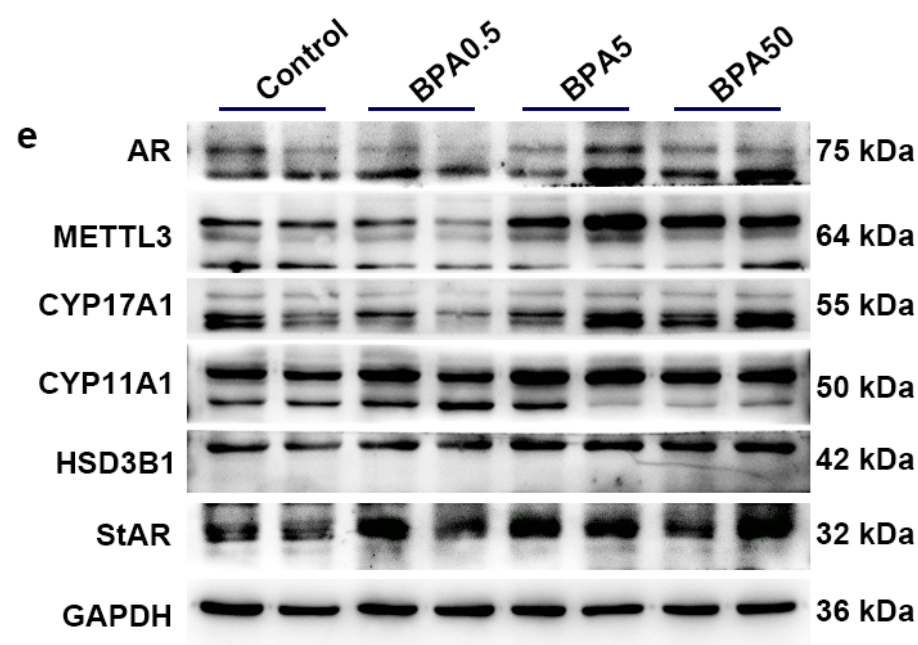

f
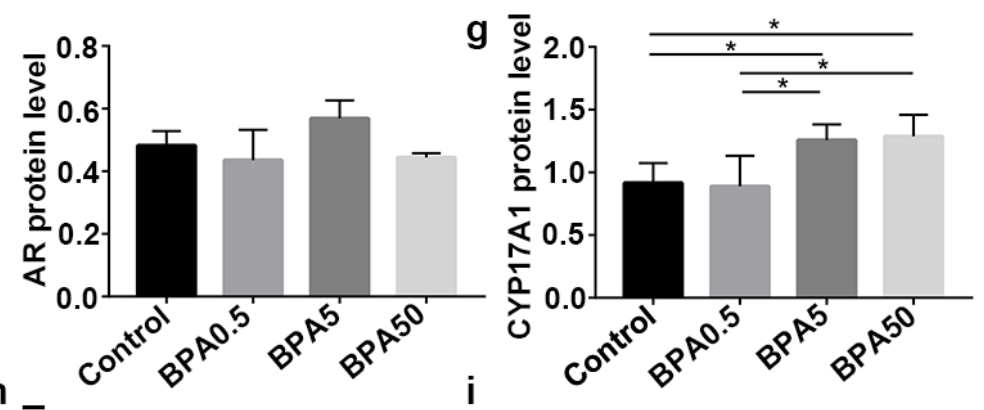

C

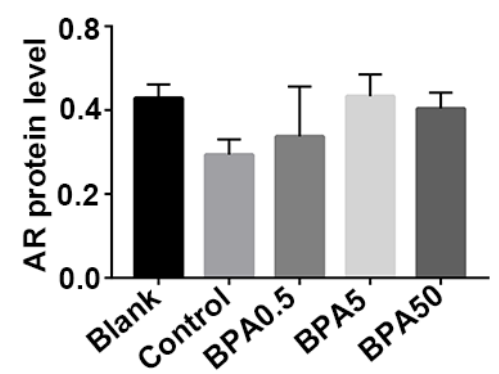

d

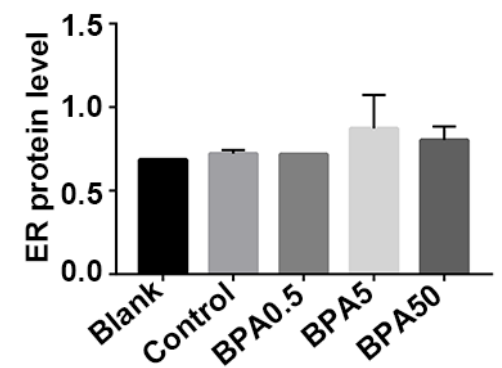
$36 \mathrm{kDa}$

h
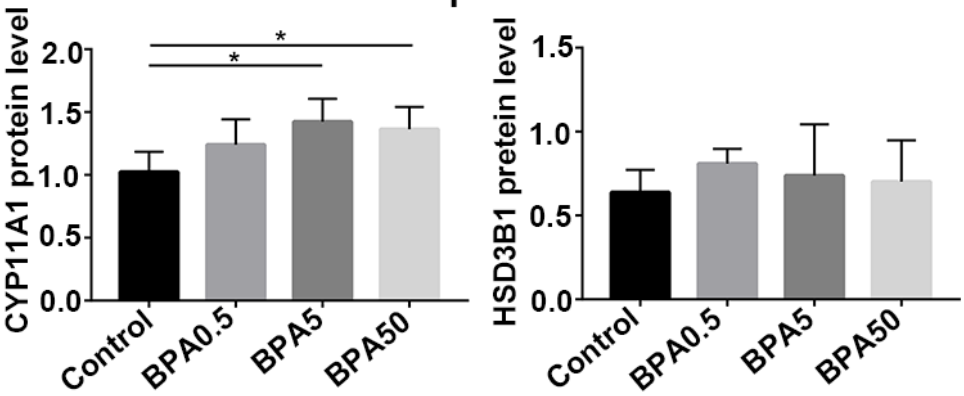

j

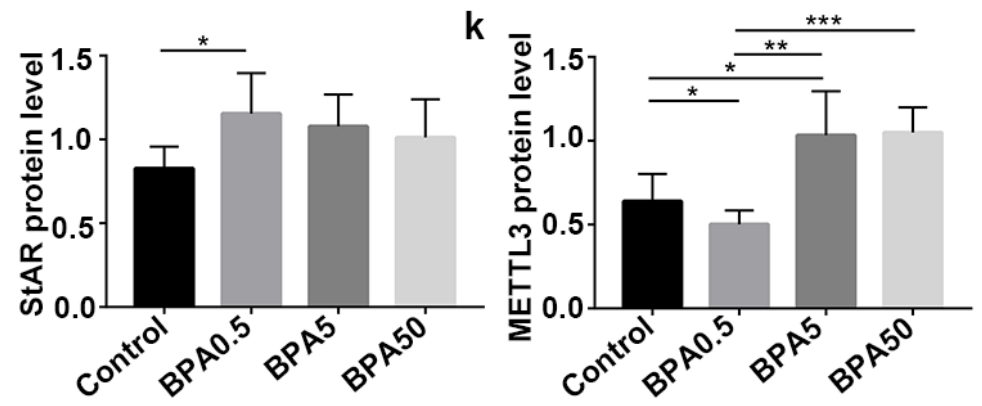

Figure 3

Bisphenol-A (BPA) disrupts the expression of androgen synthases and METTL3 without modulating hyperandrogenism. Rats received daily gavaging with BPA for 10 consecutive days. a Serum was collected from inferior vena cava. The levels of serum testosterone were measured by enzyme-linked immunosorbent assay (ELISA). * $P<0.05$. b-d Total proteins of liver were extracted using an RIPA lysis buffer. Androgen receptor and estrogen receptor molecules in livers were detected using Western blotting 
(b). The relative levels of androgen receptor (c) and estrogen receptor (d) were quantified. $\mathrm{e}-\mathrm{j}$ Total ovarian proteins were extracted using an RIPA lysis buffer. The androgen synthase and METTL3 were revealed using Western blotting (e), and relative levels of androgen receptor (f), CYP17A1 (g), CYP11A1 (h), HSD3B1 (i), StAR (j), METTL3 (k) were quantified. * $P<0.05, * * P<0.01$. 